\title{
A Method for Calculating Longitudinal Phase Space Distribution

\author{
when Given the Time Profile of the Bunch
}

Cheng-Yang Tan

Beams Division/Tevatron

\begin{abstract}
We will show in this paper a method for calculating the longitudinal phase space distribution when the time profile of the bunch as measured by a wall current monitor is given. The key to this method is the assumption that the bunch is matched to the bucket. With this assumption, we will show that the method boils down to solving a simple upper triangular matrix equation. We will also illustrate the method with two examples and show the method's shortcomings.
\end{abstract}




\section{INTRODUCTION}

The motivation of this method arose from whether there is a better way to calculate the longitudinal emittance without assuming that that the bunch is bi-gaussian. M. Church had posed this question to me and he thought that it may be possible to calculate the longitudinal phase space distribution if it is assumed that the bunch is matched to the bucket and thus obtain the emittance. After a quick library search, we found a paper by $\mathrm{Ng}^{1}$ et al who has basically worked out all the transformations needed to make this method work. In effect, this paper is an extension of their work.

In the following sections, we will first define what we mean by the cryptic phrase matched to the bucket. Then we will show how we will use Ng's transformations to work out the projected density seen on wall current monitors. From here, we will show how we can derive a matrix equation which is upper triangular and from its solution calculate the longitudinal phase space distribution. Once we have the distribution, we will show that it is easy to calculate the longitudinal emittance. Finally, we will work out two examples and show the errors and pathologies of this method. 


\section{THEORY}

In this section, we will start by defining what we mean by the phrase phase space density $\rho$ is matched to the bucket. Using this definition, we will show that $\rho$ must be a function of the Hamiltonian $\mathcal{H}$ By using a transformation from $\mathrm{Ng}$ which maps the constant $\mathrm{calH}$ contours to circles, we will show that $\mathcal{H}$ has a very simple expression in polar coordinates. This will enable us to find expressions for the radial and azimuthal phase space densities and show that only the radial density contains $\rho$. Finally, we will find an expression for the projected density and show that we can easily find $\rho$ from it. This will, of course, enable us to calculate the emittance without assuming anything about $\rho$ except that it matches the bucket.

All the phase space densities that will be discussed are normalized to 1 .

Definition of "Phase Space Density is Matched to the Bucket"

We need to define what we mean by the phrase phase space density is matched to the bucket. If the phase space density of particles $\rho$ is a constant of the motion then from the continuity equation

$$
\frac{d \rho}{d t}=\frac{\partial \rho}{\partial t}+\nabla \cdot j=0
$$

where $\boldsymbol{j}$ is the current density. Furthermore, since $\rho$ is stationary, it does not have any explicit time dependence which means that $\nabla \cdot \boldsymbol{j}=0$. For Hamiltonian systems, the contour lines in phase space do not cross, thus we can demand that

$$
\nabla \cdot \boldsymbol{j}=\nabla \cdot(\tilde{\rho}(s) \boldsymbol{v}(s))=0
$$

along a contour $\boldsymbol{s}$, where $\tilde{\rho}(s)$ is the phase space density along this contour and $\boldsymbol{v}$ is the velocity of the particle at $s$. To complete the definition of $\tilde{\rho}(s)$, the number of particles on length $d s$ is $\tilde{\rho}(s) d s$. (2) is thus our definition for "matched to the bucket". 


\section{Normalized Hamiltonian}

We will be using Ng's normalized Hamiltonian ${ }^{\dagger}$

$$
\mathcal{H}(p, \phi ; \vartheta)=\frac{1}{2} p^{2}+(1-\cos \phi)
$$

where $p, \phi$ are the conjugate momentum and position respectively and $\vartheta$ is the independent time variable which advances by $2 \pi$ every small amplitude synchrotron period. Hamilton's equations in these coordinates are

$$
\left.\begin{array}{l}
\frac{d \phi}{d \vartheta}=p \\
\frac{d p}{d \vartheta}=-\nu_{s} \sin \phi
\end{array}\right\}
$$

$\mathrm{Ng}$ has also found a set of coordinates where the contours of $\mathcal{H}$ are circles. By letting $q=2 \sin \phi / 2$, we have from (3)

$$
\mathcal{H}(p, q)=\frac{1}{2}\left(p^{2}+q^{2}\right)
$$

which are indeed circles in $(p, q)$ space. This then allow us to use polar coordinates

$$
\left.\begin{array}{l}
p=r \cos \theta \\
q=r \sin \theta
\end{array}\right\}
$$

so that $\mathcal{H}$ can be trivially written as

$$
\mathcal{H}=r^{2} / 2
$$

(Note that $\mathrm{Ng}$ had defined $\theta$ starting from the $p$-axis rather than from the $q$ axis). In this coordinate system we can show that if $\rho(p, \phi)$ is matched to the bucket, then it can be partitioned into a radial and azimuthal piece with the azimuthal piece independent of $\rho(p, \phi)$. This will then lead us to the conclusion that $\rho(p, \phi)$ must be a function of $\mathcal{H}$.

$\dagger$ We will show that Ng's normalized Hamiltonian is equivalent to the usual Edwards' normalized Hamiltonian in the Appendix. 


$$
\rho(p, \phi) \text { must be a function of } \mathcal{H}
$$

We will show that when $\rho(p, \phi)$ (the associated volume element is $d p d \phi)$ is matched to the bucket, it must be a function of $\mathcal{H} .^{\ddagger}$ From the definition of matched, if we define $\tilde{\rho}(s)$ to be the density along a contour and $\boldsymbol{v}(s)$ to be the velocity along a contour we have

$$
\nabla \cdot(\tilde{\rho}(s) \boldsymbol{v}(s))=0
$$

This means that

$$
\tilde{\rho}(s)=\frac{k(h)}{v(s)}=k(h) \frac{d \vartheta}{d s}
$$

where $k$ is a function of $\mathcal{H}=h=$ constant on a contour of constant $\mathcal{H}$. The line element $d s$ is related to $d p$ and $d \phi$ by

$$
d s=\sqrt{d p^{2}+d \phi^{2}}
$$

and on the contour, $\mathcal{H}=h$ is constant which means from (4) that

$$
h=\frac{1}{2} p^{2}+(1-\cos \phi)
$$

Let $\varrho_{\theta}(h, \theta)$ be the density on the circle of radius $h$ as shown in Figure 1 . Then it is clear from here that $\tilde{\rho}(s)$ is related to $\varrho_{\theta}(h, \theta)$ by

$$
\begin{aligned}
\tilde{\rho}(s) d s & =\varrho_{\theta}(h, \theta) d \theta \\
\Rightarrow \quad \varrho_{\theta}(h, \theta) & =\tilde{\rho}(s) \frac{d s}{d \theta}=\tilde{\rho}(s) \frac{d s}{d \vartheta} \frac{d \vartheta}{d \theta}
\end{aligned}
$$

By using the transformations previously discussed in Normalized Hamiltonian, Hamilton's equations, the chain rule, (9), (10) and (11), we can in fact show that

$$
\frac{d \theta}{d \vartheta}=\sqrt{1-\frac{h}{2} \sin ^{2} \theta}
$$

$\ddagger \overline{\text { For example, a bi-gaussian }}$ density $\rho(p, q)$ expressed in $(p, q)$ and $\mathcal{H}=\left(p^{2}+q^{2}\right) / 2$ is

$$
\rho(p, q) d p d q=A \exp \left[-\frac{1}{2 a^{2}}\left(p^{2}+q^{2}\right)\right] d p d q=A \exp \left[-\mathcal{H} / a^{2}\right] d p d q=\rho(\mathcal{H}) d p d q
$$

which is what we mean by $\rho$ being a function of $\mathcal{H}$. Density functions must always be coupled with volume elements in order to know what they refer to. 
and thus

$$
\varrho_{\theta}(h, \theta)=\frac{k(h)}{\sqrt{1-\frac{h}{2} \sin ^{2} \theta}}
$$

Therefore, on each circle all the $\theta$ dependence is in $\varrho_{\theta}$, which means that the density $\varrho_{r}$ in the $r$ direction can only have an $r$ dependence, hence $\rho(r, \theta)$ is

$$
\left.\begin{array}{rl}
\rho(r, \theta) r d r d \theta & =\varrho_{r}(r) \varrho_{\theta}\left(\frac{r^{2}}{2}, \theta\right) d r d \theta \\
& =\frac{\varrho_{r}(r) k\left(\frac{r^{2}}{2}\right)}{\sqrt{1-\frac{r^{2}}{4} \sin ^{2} \theta}} d r d \theta \\
\Rightarrow \quad \rho(p, q) d p d q & =\frac{\varrho_{r}(\sqrt{2 \mathcal{H}}) k(\mathcal{H})}{\sqrt{2 \mathcal{H}} \sqrt{1-\frac{q^{2}}{4}}} d p d q \\
\Rightarrow \quad \rho(p, \phi) d p d \phi & =\frac{\varrho_{r}(\sqrt{2 \mathcal{H}}) k(\mathcal{H})}{\sqrt{2 \mathcal{H}}} d p d \phi
\end{array}\right\}
$$

where we have used the Jacobian which relates the volume elements $d p d \phi=d p d q / \sqrt{1-\frac{q^{2}}{4}}$ in the last line. This means that $\rho(p, \phi)$ must be a function of $\mathcal{H}$ as required.

\section{Projected Density}

Referring to Figure 1, we see that the length $d q$ projected by $d \theta$ on the $q$-axis is

$$
\begin{aligned}
d q & =r \sin (\theta+d \theta)-r \sin \theta \\
& =r \cos \theta d \theta
\end{aligned}
$$

The number of particles $d n$ in $d q$ must be equal to the number of particles in $d \theta$, therefore

$$
d n=\varrho_{\theta}\left(\frac{r^{2}}{2}, \theta\right) d \theta=\frac{\varrho_{\theta}\left(\frac{r^{2}}{2}, \theta\right)}{r \cos \theta} d q=\frac{\varrho_{\theta}\left(\frac{r^{2}}{2}, \theta\right)}{\sqrt{r^{2}-q^{2}}} d q
$$

Hence, the projected density $d \rho_{\downarrow}(r, q)$ on the $q$-axis from contours in the range $r$ to $r+d r$ is

$$
d \rho_{\downarrow}(r, q)=2 u\left(r^{2}-q^{2}\right) \frac{\varrho_{r}(r) \varrho_{\theta}\left(\frac{r^{2}}{2}, \sin ^{-1}\left(\frac{q}{r}\right)\right)}{\sqrt{r^{2}-q^{2}}} d r
$$




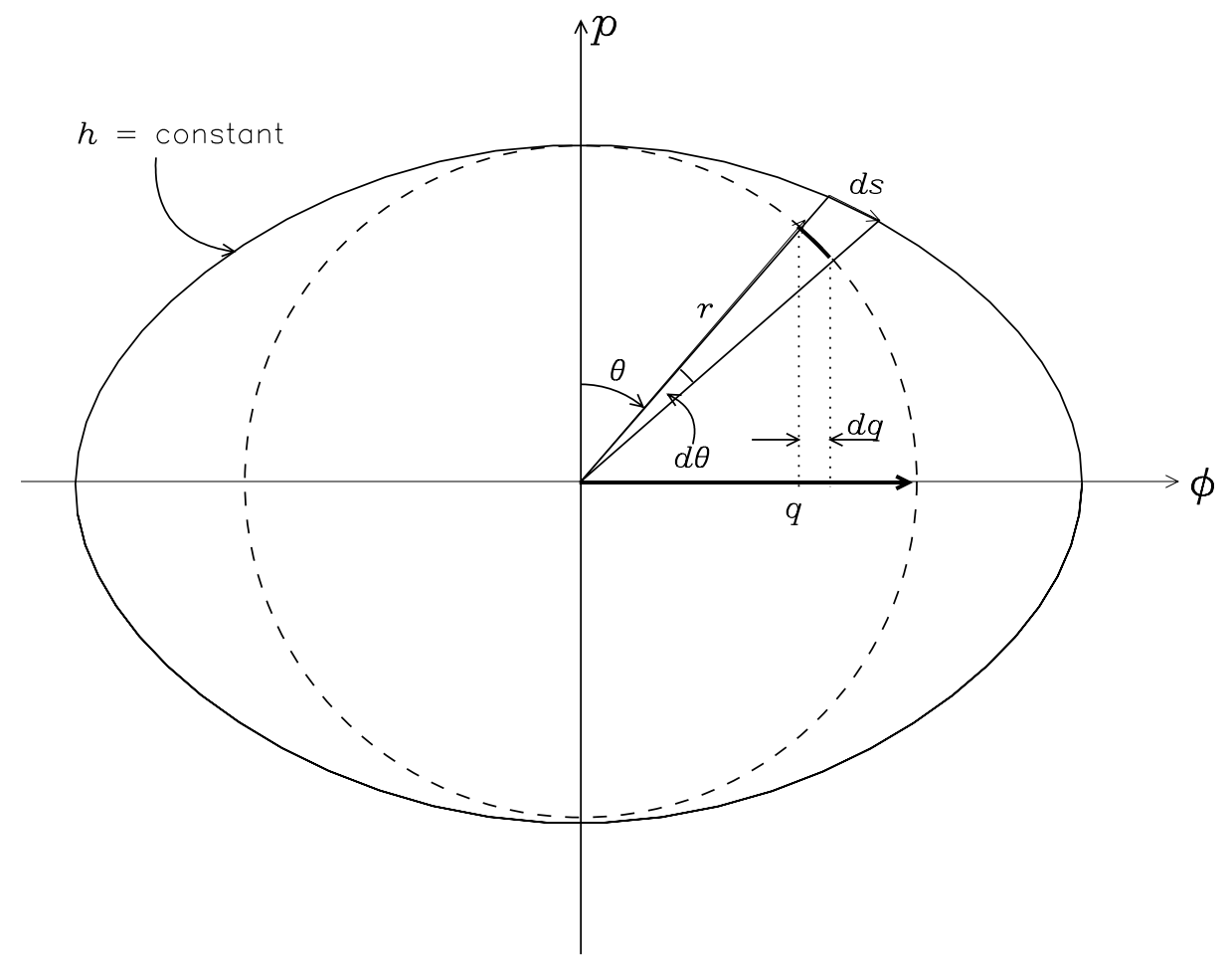

Figure 1 This shows an ellipse of constant $h$ in $(p, \phi)$ space transformed into a circle in $(r, \theta)$ space.

where the factor of 2 comes from projecting particles from upper and lower half planes, the step function $u\left(r^{2}-q^{2}\right)$ is used to ensure that the density is zero when $q>r$.

The projected density $\rho_{\downarrow}(q)$ comes from adding all the projected densities $d \rho_{\downarrow}$ of all the contours and is therefore

$$
\rho_{\downarrow}(q)=2 \int_{0}^{2} d r \varrho_{r}(r) u\left(r^{2}-q^{2}\right) \frac{\varrho_{\theta}\left(\frac{r^{2}}{2}, \sin ^{-1}\left(\frac{q}{r}\right)\right)}{\sqrt{r^{2}-q^{2}}}
$$

where the upper limit of 2 in the integral comes from the normalized Hamiltonian of (3) because $\mathcal{H}$ attains its maximum value on the contour which passes through $p=0$ and $\cos \phi=-1$. 
From (14) and (15), we have

$$
\left.\begin{array}{c}
\varrho_{r}(r)=\frac{r \rho(H(r))}{k\left(\frac{r^{2}}{2}\right)} \\
\varrho_{\theta}\left(\frac{r^{2}}{2}, \theta\right)=\frac{k\left(\frac{r^{2}}{2}\right)}{\sqrt{1-\frac{r^{2}}{4} \sin ^{2} \theta}}
\end{array}\right\}
$$

Our expressions in (20) are identical with $\mathrm{Ng}$ 's solution, when $k\left(r^{2} / 2\right) \equiv 1 /\left[4 K\left(r^{2} / 4\right)\right]$ where $K(r)$ is the complete elliptic function of the first kind as defined by Mathematica. Note: $\mathrm{Ng}^{\prime}$ s definition of $K$ is different from Mathematica.

Substituting (20) into (19), we see that

$$
\rho_{\downarrow}(q)=2 \int_{0}^{2} d r \frac{r \rho(\mathcal{H}(r)) u\left(r^{2}-q^{2}\right)}{\sqrt{\left(1-\frac{q^{2}}{4}\right)\left(r^{2}-q^{2}\right)}}
$$

is the desired result for the projected phase space density.

Check

As a check of $\rho_{\downarrow}(q)$, when we integrate $\rho_{\downarrow}(q)$ from $q=-2$ to $q=2$ the result must be 1, i.e. the following integral

$$
\int_{-2}^{2} d q \rho_{\downarrow}(q)=4 \int_{0}^{2} d r \int_{0}^{r} d q \frac{r \rho(\mathcal{H}(r))}{\sqrt{\left(1-\frac{q^{2}}{4}\right)\left(r^{2}-q^{2}\right)}}
$$

must be 1 . We note that

$$
\left.\begin{array}{rl}
\int_{0}^{r} d q \frac{1}{\sqrt{\left(1-\frac{q^{2}}{4}\right)\left(r^{2}-q^{2}\right)}} & =\frac{2}{r} F\left(\sin ^{-1} \frac{r}{2}, \frac{4}{r^{2}}\right) \\
& =K\left(\frac{r^{2}}{4}\right)
\end{array}\right\}
$$


from Mathematica where $F(x, y)$ is the elliptic integral of the first kind. Therefore $(22)$ becomes

$$
\begin{aligned}
\int_{-2}^{2} d q \rho_{\downarrow}(q) & =4 \int_{0}^{2} d r r \rho(\mathcal{H}(r)) K\left(\frac{r^{2}}{4}\right) \\
& =\int_{0}^{2} d r \varrho_{r}(r) \\
& =1
\end{aligned}
$$

by using (20).

$$
\text { Calculating } \rho(\mathcal{H}(r)) \text { from } \rho_{\downarrow}(q)
$$

Equation (21) is the key for calculating $\rho(\mathcal{H}(r))$ when we have $\rho_{\downarrow}(q)$ which is obtained from measurement. First, we need to discretize (21)

$$
\rho_{\downarrow}\left(q_{k}\right)=2 \sum_{j=1}^{N} \frac{\delta r r_{j} \rho_{j} u\left(r_{j}^{2}-q_{k}^{2}\right)}{\sqrt{\left(1-\frac{q_{k}^{2}}{4}\right)\left(r_{j}^{2}-q_{k}^{2}\right)}}
$$

where $\rho_{j}=\rho\left(\mathcal{H}\left(r_{j}\right)\right), r_{j}=j \delta r, \delta r=2 /(N+1), k=1, \ldots, M$ and $M \geq N$. This equation means that we can write a set of linear equations in matrix form

$$
w_{i}=M_{i j} R_{j}
$$

where

$$
\left.\begin{array}{rl}
w_{i} & =\rho_{\downarrow}\left(q_{i}\right) \\
M_{i j} & =\frac{2 u\left(r_{j}^{2}-q_{i}^{2}\right)}{\sqrt{\left(1-\frac{q_{k}^{2}}{4}\right)\left(r_{j}^{2}-q_{i}^{2}\right)}} \\
R_{j} & =r_{j} \rho_{j} \delta r
\end{array}\right\}
$$

If $M=N$ and $r_{j}=q_{j}$ then $M_{i j}$ is actually upper triangular i.e.

$$
\boldsymbol{M}=\left(\begin{array}{ccccc}
M_{11} & M_{12} & M_{13} & \ldots & M_{1 N} \\
0 & M_{22} & M_{23} & \ldots & M_{2 N} \\
\vdots & \vdots & \ddots & \ldots & \vdots \\
0 & 0 & \ldots & M_{N-1, N-1} & M_{N-1, N} \\
0 & 0 & \ldots & 0 & M_{N N}
\end{array}\right)
$$


because of $u\left(r_{j}^{2}-q_{i}^{2}\right)$ which makes for solving $R_{j}$ a trivial exercise. If $M>N$, then we have an over-constrained system which means that we have to use least square methods to ensure a solution.

\section{Calculating Emittance}

To calculate the emittance from $R_{j}$, what we basically need to do is find $r_{\mu}$ such that

$$
\int_{0}^{r_{\mu}} \int_{0}^{2 \pi} \varrho_{r}(r) \varrho_{\theta}(r, \theta) d r d \theta=\mu \quad \text { where } 0 \leq \mu \leq 1
$$

which involves a double integral, or even better use (24)

$$
4 \int_{0}^{r_{\mu}} d r r \rho(\mathcal{H}(r)) K\left(\frac{r^{2}}{4}\right)=\mu \quad \text { where } 0 \leq \mu \leq 1
$$

which only involves a single integral. In practice, we will probably set $\mu=0.95$. (30) when discretized and written in terms of $R_{j}$ is

$$
4 \sum_{i=1}^{k} R_{i} K\left(\frac{r_{\mu, i}^{2}}{4}\right)<\mu<4 \sum_{i=1}^{k+1} R_{i} K\left(\frac{r_{\mu, i}^{2}}{4}\right)
$$

where $r_{\mu, i}=i \delta r, \delta r=2 /(N+1)$.

With $r_{\mu, k}$ we can calculate $\mathcal{H}_{\mu}=r_{\mu, k}^{2} / 2$ and from here the emittance $\epsilon$ is easily calculated using

$$
\epsilon=2 \int_{\phi_{-}}^{\phi_{+}} d \phi \sqrt{r_{\mu, k}^{2}-2(1-\cos \phi)}
$$

where $\phi_{ \pm}= \pm \cos ^{-1}\left(1-r_{\mu, k}^{2} / 2\right)$. The following equation will convert $\epsilon$ to the usual units

$$
\varepsilon(\mathrm{eV} \cdot \mathrm{s})=\frac{\epsilon \beta}{\omega_{R F}} \sqrt{\frac{e V E_{s}}{2 \pi h|\eta|}}
$$

\section{Caveats}

In $(r, \theta)$ space we have a degeneracy for calculating $\rho_{j}$ if $r_{j}$ is ever zero because $R_{j}=$ $r_{j} \rho_{j} \delta r$ means that $\rho_{j}$ is indeterminate at $r_{j}=0$. In practice, although we will never use 
$\rho_{j}$ when $r_{j}=0$, the problem is that for small $r_{j}, R_{j}$ will be dominated by $r_{j}$ and not $\rho_{j}$ which means that our method is insensitive to perturbations near zero, i.e. it will yield an incorrect answer for $\rho_{j}$ near zero. However, if we are only interested in $r_{j} \rho_{j}$ for determining $\epsilon$, then this is not a problem. 


\section{EXAMPLES}

We will look at two examples in this section. The first example is the bi-gaussian distribution given by

$$
\rho(p, \phi)=A \exp \left[-\frac{1}{2 a^{2}}\left(p^{2}+4 \sin ^{2} \frac{\phi}{2}\right)\right]
$$

We will choose $a=0.5$ and set $A=0.6137977$ so that $\rho(p, \phi)$ is normalized to 1 . Figure 2 shows $\rho_{\downarrow}$ for the bi-gaussian distribution.

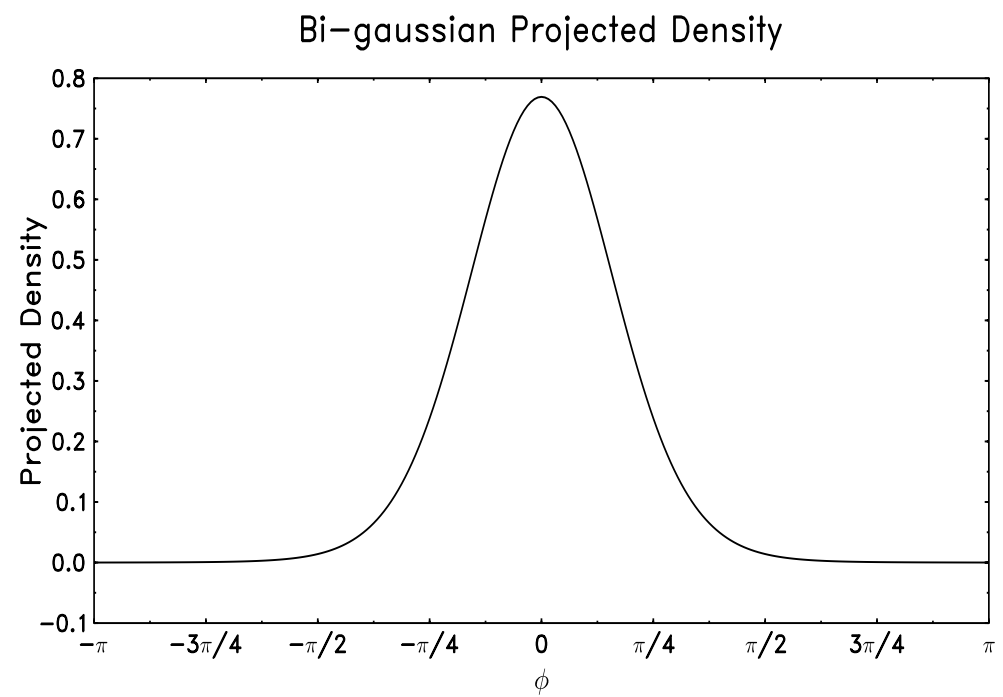

Figure 2 This is the projected phase space density on the $\phi$ axis of the bi-gaussian distribution used in our example.

Since clearly $\rho_{\downarrow}$ is symmetric about $q=0$, we can sample from $q=0$ to $q=2$ with $N$ points and then use $(26),(27)$ to calculate $R$. We can then easily compare $R / \delta r$ thus calculated with the exact curve which is given by

$$
R_{\text {exact }}(r) / \delta r=A r \exp \left[-\frac{r^{2}}{2 a^{2}}\right]
$$




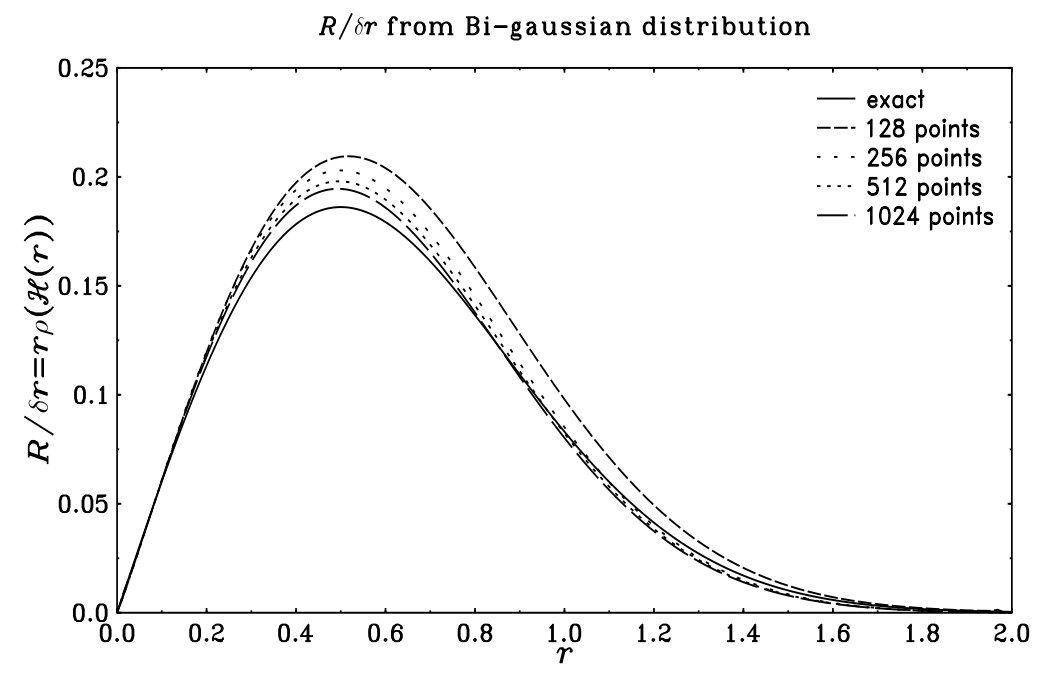

Figure 3 This figure shows the calculated $R / \delta r$ when $N=128$, 256,512 and 1024 and the exact solution. We see that $R / \delta r$ converges slowly towards the exact solution as $N$ increases.

Figure 3 shows the results for the cases when $N=128,256,512$ and 1024 and we see that convergence is rather slow.

Next, we can calculate $r$ s.t. $\mu=0.95$ and compare it with the exact solution. But before we do this, we have to realize that

$$
\text { norm }=4 \sum_{i=1}^{N} R_{i} K\left(\frac{r_{0.95, i}^{2}}{2}\right)>1
$$

because all the calculated $R / \delta r$ curves enclose a bigger area than the exact solution. However, this can be easily remedied by renormalizing (36) to 1 . If we do this, we can produce Table 1 which shows that the error between the exact and calculated solutions differ by $4 \%$. In Table 1, we have introduced another symbol $r_{(N)}$ which represents the calculated radius when $\mu=0.95$ and the number of sampling points is $N$. 


\begin{tabular}{c|c|c}
\hline \multicolumn{3}{c}{ Table 1. $r_{\text {exact }}=1.25$ for ex. $\mathbf{1}$} \\
\hline $\boldsymbol{N}$ & norm & $\boldsymbol{r}_{(\boldsymbol{N})}$ \\
128 & 1.09 & 1.20 \\
256 & 1.053 & 1.203 \\
512 & 1.026 & 1.203 \\
1024 & 1.007 & 1.203 \\
\hline \hline
\end{tabular}

The difference between the $N=1024$ calculated curve and the exact curve is plotted in Figure 4. We see the reason why the calculated $r_{(1024)}<r_{(\text {exact })}$ because for $r<1.2$, the summation using $\left(R_{1024} / \delta r\right) /$ norm will always be larger than when using $R_{\text {exact }} / \delta r$. Note that the integral of the error from $0<r<2$ is zero.

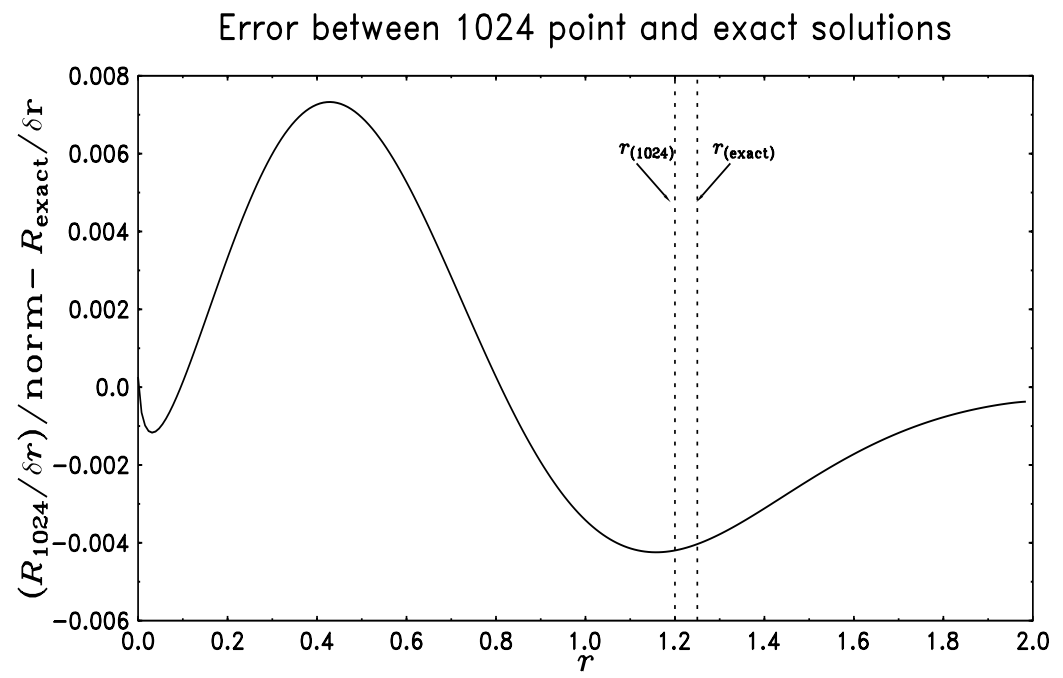

Figure 4 This figure shows the difference between the $N=1024$ calculated curve and the exact curve. Shown also is $r_{(1024)}=1.2$ and $r_{(\text {exact })}=1.25$. 


\section{Example 2}

In this example we will examine an annulus distribution in phase space

$$
\rho(p, \phi)= \begin{cases}A\left(p^{2}+4 \sin ^{2} \frac{\phi}{2}\right) & \text { if } \frac{1}{2}<\frac{1}{2}\left(p^{2}+4 \sin ^{2} \frac{\phi}{2}\right)<1 \\ 0 \quad \text { otherwise } & \end{cases}
$$

And $A=0.188011$ so that $\rho$ is normalized to 1 . This distribution is rather contrived and its main purpose is to show some pathologies of our method. The projected distribution $\rho_{\downarrow}$ looks like batman which is shown in Figure 5 . We can calulate $R / \delta r$ for the cases when the number of sampling points $N=128,256,512$ and 1024 between $q=0$ and $q=2$. Doing so, we can plot the results in Figure 6 with the exact solution and see that there are two pathological behaviours

(i) At the edges of $R / \delta r$, the calculated $R / \delta r$ have sharp spikes. Worse, at the left edge, $R / \delta r$ actually goes negative, when we know that $R / \delta r \geq 0$ for $0 \leq r \leq 2$.

(ii) As $N$ increases, the calculated $R / \delta r$ fails to converge to the exact solution which is given by

$$
R_{\text {exact }}(r) / \delta r= \begin{cases}A r^{3} & \text { if } 1<r<\sqrt{2} \\ 0 & \text { otherwise }\end{cases}
$$

Clearly, we have to be able to identify these two pathologies when given any arbitrary $\rho_{\downarrow}$ which matches the bucket. Case $(i)$ is easily identified by looking at $R(r)$. Case (ii) is identified when we perform the summation shown below and find that it is $<1$.

$$
\operatorname{norm}=4 \sum_{i=1}^{N} R_{i} K\left(\frac{r_{0.95, i}^{2}}{2}\right)<1
$$

Both cases are not showstoppers for practical implementations, but more work needs to be done to understand why these pathologies occur and to truly fix the algorithm.

Disregarding the pathologies for the moment, we will perform the exact same renormalization to (39) as in the previous example in order to calculate $r$ for $\mu=0.95$ and 


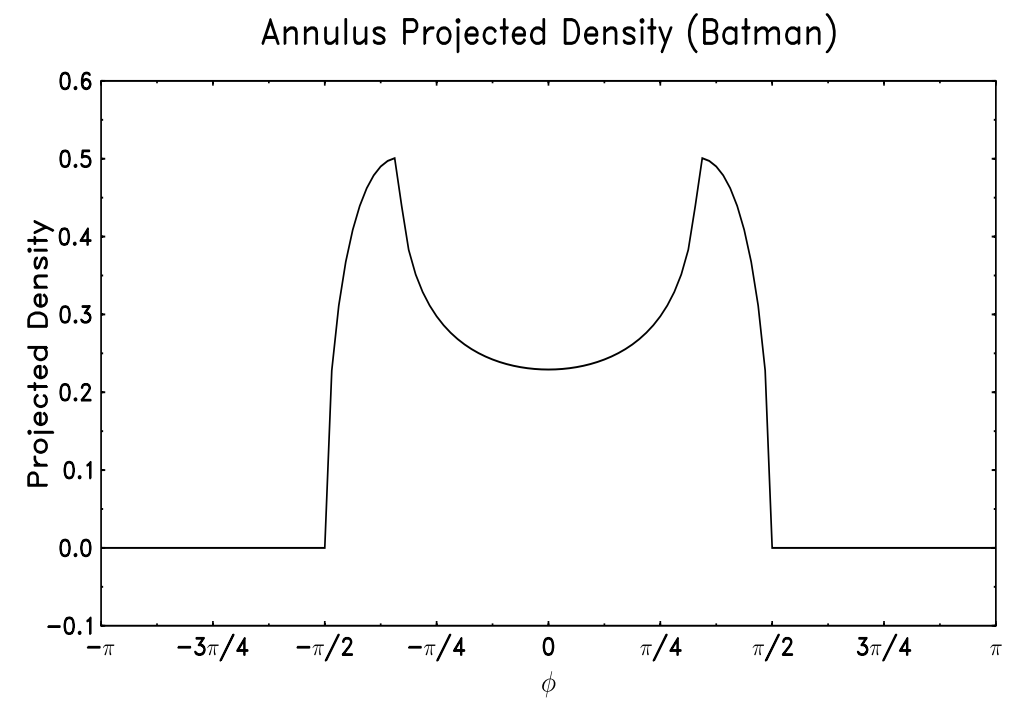

Figure 5 This is the projected phase space density on the $\phi$ axis of an annulus (batman) distribution.

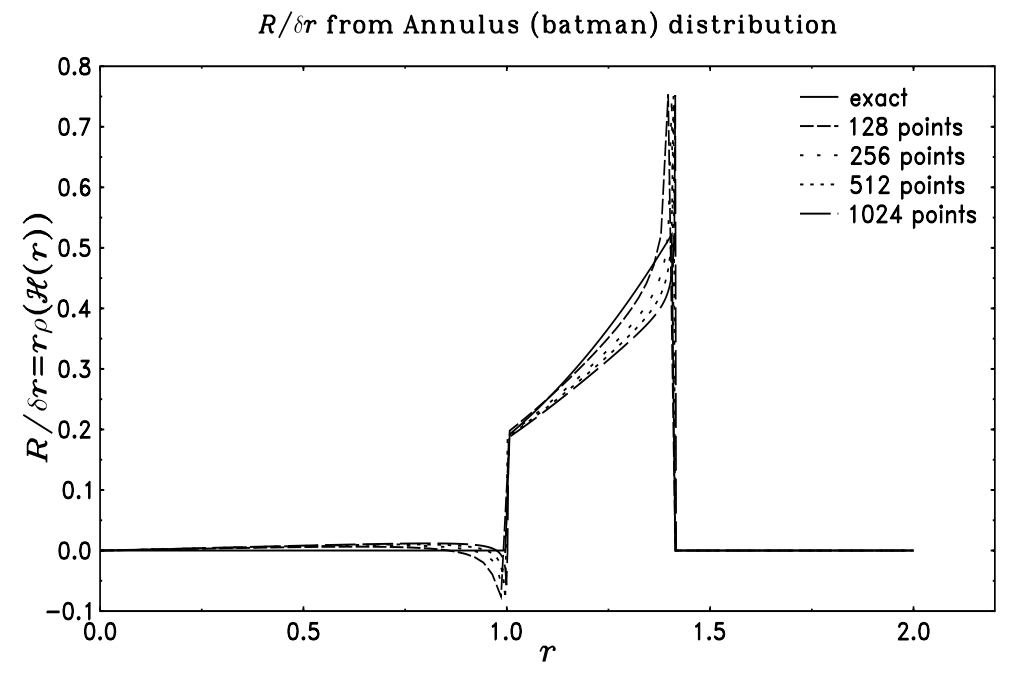

Figure 6 This figure shows the calculated $R / \delta r$ when $N=128$, 256, 512 and 1024 and the exact solution. We see two pathologies here which are discussed in the text. 
compare it with the exact solution. This is shown in Table 2 which shows that the error between the exact and calculated solutions are small once renormalization has been carried out. This outcome should not be surprising because

( $i$ ) we have cheated by fixing the summation by normalizing it.

(ii) $R / \delta r$ only occupies a small fraction of the range of $r$, in fact only between 1 and $\sqrt{2}$, thus $95 \%$ must be close to $\sqrt{2}$ after normalization.

\begin{tabular}{c|c|c}
\hline \multicolumn{3}{c}{ Table 2. $r_{\text {exact }}=1.40$ for ex. 2 } \\
\hline $\boldsymbol{N}$ & norm & $\boldsymbol{r}_{(\boldsymbol{N})}$ \\
128 & 0.97 & 1.38 \\
256 & 0.947 & 1.397 \\
512 & 0.929 & 1.397 \\
1024 & 0.916 & 1.399 \\
2048 & 0.907 & 1.401 \\
\hline \hline
\end{tabular}

CONCLUSION

By using the previous two examples, we have shown how we can calculate the longitudinal phase space distribution when it is given that the bunch is matched to the bucket. For the bigaussian distribution, the method works but convergence is slow. For the batman distribution, there are problems with this method which need to be handled in a practical implementation. We believe that these problems can be fixed if we put in the constraints that $R_{i}>0$ and $4 \sum_{i} R_{i} K\left(\frac{r_{\mu, i}^{2}}{4}\right)=1$. 


\section{APPENDIX}

We start with the usual normalized Hamiltonian from Edwards ${ }^{2}$ which describes a stationary bucket

$$
\left.\begin{array}{rl}
\mathcal{H}(p, \varphi ; n) & =\frac{p^{2}}{2}+\lambda^{2} \cos \varphi \\
\lambda^{2} & =\frac{2 \pi h \eta e V}{\beta^{2} E_{s}}
\end{array}\right\}
$$

where we have assumed that we are above transition, i.e. $\eta>0$ and the synchronous phase is $\pi$ and have used symbols with their usual meanings.

Let's make a change of variables $\varphi=\pi+\phi$, so that the bucket is now centred at $\phi=0$,

$$
\mathcal{H}(p, \phi ; n)=\frac{p^{2}}{2}-\lambda^{2} \cos \phi
$$

We will transform (41) to the one used by $\mathrm{Ng}$. First, our normalized $p$ is related to $\Delta E / E_{s}$ by Edwards (2.39)

$$
\left.\begin{array}{l}
\frac{d \phi}{d n}=p=2 \pi \eta h\left(\frac{1}{\beta^{2}} \frac{\Delta E}{E_{s}}\right) \equiv 2 \pi \eta h \delta \\
\frac{d \phi}{d \vartheta}=p^{\prime}=\eta h \delta
\end{array}\right\}
$$

where we have used Hamilton's equation and defined $\vartheta=2 n \pi$. The other Hamilton's equation gives

$$
\left.\begin{array}{rl}
\frac{d p}{d n} & =-\lambda^{2} \sin \phi \\
\Rightarrow \quad \frac{d p^{\prime}}{d n} & =-\frac{h \eta e V}{\beta^{2} E_{s}} \sin \phi \\
\Rightarrow \quad \frac{d p^{\prime}}{d \vartheta} & =-\frac{h \eta e V}{2 \pi \beta^{2} E_{s}} \sin \phi \equiv-\nu_{s}^{2} \sin \phi
\end{array}\right\}
$$

Finally, by defining Ng's normalized momentum to be $p^{\prime \prime}=p^{\prime} / \nu_{s}$, and substituting it into (42) and (43) we have the following Hamilton's equations

$$
\left.\begin{array}{rl}
\frac{d \phi}{d \vartheta} & =\nu_{s} p^{\prime \prime} \\
\frac{d p^{\prime \prime}}{d \vartheta} & =-\nu_{s} \sin \phi
\end{array}\right\}
$$


which is exactly what $\mathrm{Ng}$ obtained. These equations then produce the normalized $\mathrm{Ng}$ Hamiltonian

$$
\mathcal{H}\left(p^{\prime \prime}, \phi ; \vartheta\right)=\frac{1}{2} \nu_{s} p^{2}+\nu_{s}(1-\cos \phi)
$$

and by changing the independent variable $\vartheta$ to $\vartheta=\nu_{s} \vartheta^{\prime}$ we finally obtain the required normalized Hamiltonian used by $\mathrm{Ng}$.

\section{ACKNOWLEDGEMENTS}

The author wishes to thank M. Church for his original idea and K.Y. Ng for fruitful discussions, and for both of them for reading the first draft of this paper.

\section{REFERENCES}

[1] Particle Population Inside a Tight Stationary Bucket, K.Y. Ng, K.M. Fung and S.Y. Lee, FN-695, August 2000.

[2] An Introduction to the Physics of High Energy Accelerators, D.A. Edwards and M.J. Syphers, Wiley Series in Beam Physics, 1993. 